\title{
Modulation of Water Exchange in Europium(III) DOTA-tetraamide Complexes via Electronic Substituent Effects
}

S. James Ratnakar, ${ }^{\ddagger}$ Mark Woods, ${ }^{\dagger}$, Angelo J.M. Lubag, ${ }^{\ddagger}$ Zoltán Kovács, ${ }^{\S \ddagger}$ and A. Dean

$$
\text { Sherry.*\$, }
$$

${ }^{\ddagger}$ Advanced Imaging Research Center, UT Southwestern Medical Center, 5323 Harry Hines Blvd, Dallas, Texas, 75390, ${ }^{\dagger}$ Macrocyclics, 2110 Research Row, Ste 425, Dallas, Texas, 75235 and ${ }^{\S}$ Department of Chemistry, University of Texas at Dallas, $800 \mathrm{~W}$. Campbell Rd, Richardson, Texas, 75080 


\section{ja076325y: Ratnakar et al. Supplementary Information S1}

\section{CEST Spectroscopy}

CEST spectra of $20 \mathrm{mM}$ solutions of the complexes in 50\% water in acetonitrile were recorded on a Varian Mercury spectrometer operating at $300 \mathrm{MHz}$. Pre-saturation pulses of $2 \mathrm{~s}$ duration were applied at $450 \mathrm{~Hz}$ (blue), $325 \mathrm{~Hz}$ (red) and $230 \mathrm{~Hz}$ (green). The black lines through each point are fits to the experimental data using the Bloch equation modified for exchange.
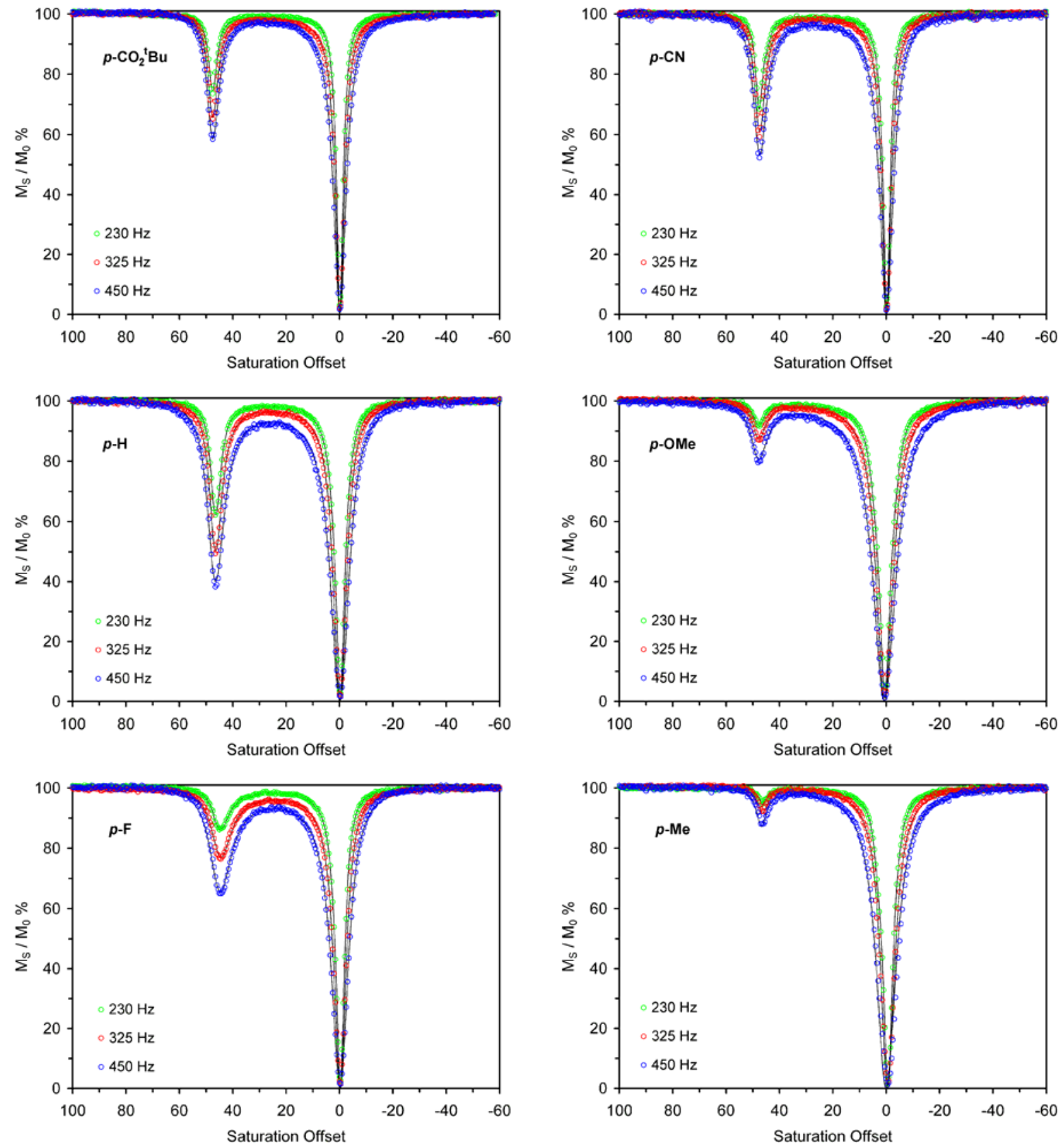


\section{ja076325y: Ratnakar et al. Supplementary Information S2}

\section{CEST Fitting}

The CEST spectra were fitted by previously published methods \{Woessner, D. E.; Zhang, S.; Merritt, M. E.; Sherry, A. D. Magn. Reson. Med. 2005, 53, 790-799, Woods, M.; Woessner, D. E.; Zhao, P.; Pasha, A.; Yang, M.-Y.; Huang, C.-H.; Vasalitiy, O.; Morrow, J. R.; Sherry, A. D. J. Am. Chem. Soc. 2006, 128, 10155-10162.\} affording the following parameters:

\begin{tabular}{c|cccc|cc}
\hline & \multicolumn{4}{|c|}{ Metal Bound water } & \multicolumn{2}{c}{ Solvent Water } \\
& $\tau_{\mathrm{M}}(\mathrm{ms})$ & $\delta(\mathrm{ppm})$ & $\mathrm{T}_{1}(\mathrm{~s})$ & $\mathrm{T}_{2}(\mathrm{~s})$ & $\mathrm{T}_{1}(\mathrm{~s})$ & $\mathrm{T}_{2}(\mathrm{~s})$ \\
\hline $\mathrm{CO}_{2}{ }^{\mathrm{t}} \mathrm{Bu}$ & 0.352 & 47.69 & 0.176 & 0.011 & 0.795 & 0.487 \\
$\mathrm{CN}$ & 0.324 & 47.61 & 0.029 & 0.011 & 1.146 & 0.910 \\
$\mathrm{~F}$ & 0.144 & 44.98 & 0.010 & 0.011 & 0.852 & 0.386 \\
$\mathrm{H}$ & 0.269 & 46.72 & 0.656 & 0.011 & 2.121 & 0.749 \\
$\mathrm{OMe}$ & 0.198 & 47.25 & 0.036 & 0.011 & 0.290 & 0.025 \\
$\mathrm{Me}$ & 0.310 & 46.89 & 0.010 & 0.011 & 0.171 & 0.020 \\
\hline
\end{tabular}




\section{ja076325y: Ratnakar et al. Supplementary Information S3}

\section{Imaging Protocol}

CEST imaging was performed on a phantom of six different $5 \mathrm{~mm}$ NMR tubes containing 10 $\mathrm{mM}$ solutions of the complexes dissolved in water and acetonitrile $(1: 1 \mathrm{v} / \mathrm{v})$. Images were acquired on a Varian Unity INOVA $400 \mathrm{MHz}$ vertical wide - bore spectrometer (89 mm), with gradients capable of 120 gauss $/ \mathrm{cm}$. A $20 \mathrm{~mm}$ imaging probe (volume coil) and the standard spin-echo pulse sequence with saturation (SEMSCONSAT) were used. A 3 s presaturation pulse $\left(\mathrm{B}_{1}=24 \mu \mathrm{T}\right)$ was employed with TR $=10 \mathrm{~s}, \mathrm{TE}=8.2 \mathrm{~ms}$ and a matrix $=128 \times 128, \mathrm{FOV}=25 \mathrm{~mm} \times 25 \mathrm{~mm}$, thickness $=5 \mathrm{~mm}$ transverse slice. Images were collected with saturation at two different frequencies: $-47.5 \mathrm{ppm}$ and at $+47.5 \mathrm{ppm}$ from the bulk water signal.

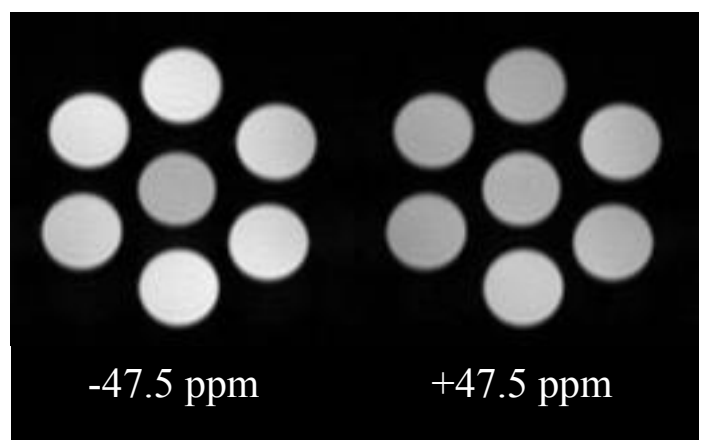

Figure S3. CEST images at two different frequencies: $-47.5 \mathrm{ppm}$ and $+47.5 \mathrm{ppm}$ from the bulk water signal.

\section{Image Processing}

The CEST images were obtained by subtracting the corresponding $-47.5 \mathrm{ppm}$ (off-resonance) image from the $+47.5 \mathrm{ppm}$ image (on bound water resonance) using the ImageJ (NIH) program. A $2 \times$ 2 median filter (replacing each pixel value with the median of the neighbouring pixel values) was applied to improve signal-to-noise in the images. 


\section{ja076325y: Ratnakar et al. Supplementary Information S4}

\section{Experimental}

General Remarks. All solvents and reagents were purchased from commercial sources and used as received unless otherwise stated. ${ }^{1} \mathrm{H}$ and ${ }^{13} \mathrm{C}$ NMR spectra were recorded on a Varian INOVA 400 spectrometer operating at 400 and $100 \mathrm{MHz}$, respectively. CEST spectra were recorded on a Varian Mercury spectrometer operating at $300 \mathrm{MHz}$. MALDI mass spectra were acquired on an Applied Biosystems Voyager-6115 mass spectrometer. Melting points were determined on a FisherJohns melting point apparatus and are uncorrected. "Water" refers to high purity water with a resistance of $>18 \mathrm{M} \Omega$.

\section{Synthesis}

The ligands were synthesized according to the general scheme shown (Scheme 1). Ethyl bromoacetamidoacetate 1 was synthesized according to literature procedures (S. Zhang, K. $\mathrm{Wu}$, M.C. Biewer, \& A.D. Sherry, Inorg. Chem. 2007, 40 (17), 4284-4290).

Tris-( $N$-ethylacetate)-1,4,7,10-tetraazacyclododecane-1,4,7triacetamide (2). Cyclen $(0.86 \mathrm{~g}, 5 \mathrm{mmol})$ and $\mathrm{NaHCO}_{3}(2.07$ $\mathrm{g}, 15 \mathrm{mmol})$ were added to acetonitrile $(100 \mathrm{~mL})$ and heated, with stirring, at $65^{\circ} \mathrm{C}$ for $1 \mathrm{~h}$. Ethyl-2-bromoacetamido acetate $1(3.36 \mathrm{~g}, 15 \mathrm{mmol})$ in acetonitrile $(20 \mathrm{~mL})$ was then added and the reaction mixture stirred at $65^{\circ} \mathrm{C}$ for $72 \mathrm{~h}$. The reaction was cooled to room temperature and filtered. The solvents were removed from the filtrate under reduced pressure and the residue purified by column chromatography over silica gel, eluting with $\mathrm{CH}_{2} \mathrm{Cl}_{2} / \mathrm{CH}_{3} \mathrm{OH}(80: 20 \mathrm{v} / \mathrm{v})$. The title compound was afforded as a pale yellow hygroscopic solid (1.22 g, 29\%). $R_{\mathrm{f}}=0.36\left(\mathrm{SiO}_{2}, 20 \% \mathrm{MeOH}\right.$ in $\left.\mathrm{CH}_{2} \mathrm{Cl}_{2}\right) ;{ }^{1} \mathrm{H} \mathrm{NMR}(400 \mathrm{MHz}$, $\left.\mathrm{D}_{2} \mathrm{O}\right), \delta=4.09\left(4 \mathrm{H}, \mathrm{q},{ }^{3} J_{\mathrm{H}-\mathrm{H}}=8 \mathrm{~Hz}, \mathrm{OCH}_{2}\right), 4.09\left(2 \mathrm{H}, \mathrm{q},{ }^{3} J_{\mathrm{H}-\mathrm{H}}\right.$ $\left.=8 \mathrm{~Hz}, \mathrm{OCH}_{2}\right), 3.87\left(6 \mathrm{H}, \mathrm{s}, \mathrm{NHCH}_{2} \mathrm{CO}_{2}\right), 3.23(2 \mathrm{H}, \mathrm{s}$, $\left.\mathrm{NCH}_{2} \mathrm{C}=\mathrm{O}\right), 3.22\left(4 \mathrm{H}, \mathrm{s}, \mathrm{NCH}_{2} \mathrm{C}=\mathrm{O}\right), 2.92-2.62(16 \mathrm{H}, \mathrm{m}$ br, ring $\left.\mathrm{CH}_{2} \mathrm{~N}\right), 1.15\left(3 \mathrm{H}, \mathrm{t},{ }^{3} \mathrm{~J}_{\mathrm{H}-\mathrm{H}}=8 \mathrm{~Hz}, \mathrm{CH}_{3}\right), 1.14\left(6 \mathrm{H}, \mathrm{t},{ }^{3} J_{\mathrm{H}-\mathrm{H}}=\right.$ $\left.8 \mathrm{~Hz}, \mathrm{CH}_{3}\right) ;{ }^{13} \mathrm{C}$ NMR $\left(100 \mathrm{MHz}, \mathrm{D}_{2} \mathrm{O}\right) \delta=174.9\left(2 \times \mathrm{CO}_{2} \mathrm{Et}\right)$, $171.7(2 \times \mathrm{NHC}=\mathrm{O}), 62.8\left(\mathrm{OCH}_{2}\right), 62.7\left(\mathrm{OCH}_{2}\right), 57.2$ $\left(\mathrm{NHCH}_{2} \mathrm{CO}_{2} \mathrm{Et}\right), 57.1\left(\mathrm{NHCH}_{2} \mathrm{CO}_{2} \mathrm{Et}\right), 52.0\left(\right.$ ring $\left.\mathrm{CH}_{2} \mathrm{~N}\right), 51.6$ (ring $\mathrm{CH}_{2} \mathrm{~N}$ ), 44.4 (ring $\mathrm{CH}_{2} \mathrm{~N}$ ), 44.3 (ring $\mathrm{CH}_{2} \mathrm{~N}$ ), 43.4 $\left(\mathrm{NCH}_{2} \mathrm{CO}\right), 41.3\left(\mathrm{NCH}_{2} \mathrm{CO}\right), 13.5\left(2 \times \mathrm{CH}_{3}\right) ; \mathrm{m} / \mathrm{z}(\mathrm{MALDI}+)$ $602(100 \%),[\mathrm{M}+\mathrm{H}]^{+}, 624\left(8 \%,[\mathrm{M}+\mathrm{Na}]^{+}\right)$. Accurate melting points and combustion analyses could not be determined for this compound owing to its hygroscopic nature.

General procedure for the synthesis of $N$-arylbromoacetamides. To a solution of $p$-substituted analine $(32.2$ $\mathrm{mmol})$ in dichloromethane $(50 \mathrm{~mL})$ was added potassium carbonate $(35.1 \mathrm{mmol})$. The reaction mixture was stirred vigorously and cooled to $0{ }^{\circ} \mathrm{C}$ in an ice bath. Bromoacetyl bromide $(35.1 \mathrm{mmol})$ in dichloromethane $(50 \mathrm{~mL})$ was then added drop-wise over a period of $1 \mathrm{hr}$. The reaction mixture was then allowed to warm to room temperature and stirred for a further $6 \mathrm{~h}$. The reaction was then quenched by cautious addition of water $(30 \mathrm{~mL})$. Methanol $(60 \mathrm{~mL})$ was added to afford a homogeneous solution and the $N$-aryl bromoacetamide isolated by slow evaporation of the solvents at room temperature.

4-Methylphenyl bromoacetamide (3). The title compound was obtained as colorless crystals (4.2 g, $72 \%) . \mathrm{Mp}=152$ $154{ }^{\circ} \mathrm{C} ;{ }^{1} \mathrm{H}$ NMR $\left(400 \mathrm{MHz}, \mathrm{CD}_{3} \mathrm{CN}\right) \delta=10.00(1 \mathrm{H}, \mathrm{s}$ br, NH), $7.16\left(2 \mathrm{H}, \mathrm{d},{ }^{3} J_{\mathrm{H}-\mathrm{H}}=8 \mathrm{~Hz}, \mathrm{Ar}\right), 6.82\left(2 \mathrm{H}, \mathrm{d},{ }^{3} J_{\mathrm{H}-\mathrm{H}}=8 \mathrm{~Hz}, \mathrm{Ar}\right)$, $3.72\left(2 \mathrm{H}, \mathrm{s}, \mathrm{BrCH}_{2}\right), 1.94\left(3 \mathrm{H}, \mathrm{s}, \mathrm{CH}_{3}\right) ;{ }^{13} \mathrm{C} \mathrm{NMR}(100 \mathrm{MHz}$, $\left.\mathrm{CD}_{3} \mathrm{CN}\right) \delta=165.2(\mathrm{C}=\mathrm{O}), 136.7$ (1-Ar), 133.5 (4-Ar), 129.9 (2Ar, 3-Ar), $31.1\left(\mathrm{BrCH}_{2}\right), 21.1\left(\mathrm{CH}_{3}\right) . \mathrm{m} / \mathrm{z}$ (MALDI+) $228(100$ $\left.\%,[\mathrm{M}+\mathrm{H}]^{+}\right)$.

4-Fluorophenyl bromoacetamide (4). The title compound was obtained as colorless crystals $(4.5 \mathrm{~g}, 84 \%) . \quad \mathrm{Mp}=137$ $138{ }^{\circ} \mathrm{C} ;{ }^{1} \mathrm{H}$ NMR $\left(400 \mathrm{MHz}, \mathrm{CD}_{3} \mathrm{CN}\right) \delta=8.76(1 \mathrm{H}, \mathrm{s}$ br, $\mathrm{NH})$, $7.57\left(2 \mathrm{H}, \mathrm{dd},{ }^{3} J_{\mathrm{H}-\mathrm{H}}=9 \mathrm{~Hz},{ }^{4} J_{\mathrm{F}-\mathrm{H}}=5 \mathrm{~Hz}, 2-\mathrm{Ar}\right), 7.09(2 \mathrm{H}, \mathrm{dd}$,<smiles>[X]c1ccc(N)cc1</smiles><smiles>[X]c1ccc(NC(=O)CN2CCN(CC(=O)NCC(C)=O)CCN(CC(=O)NCC(=O)NCC(C)=O)CCN(CC(=O)NCC(=O)NCC(C)=O)CC2)cc1</smiles>

Scheme 1. 


\section{ja076325y: Ratnakar et al. Supplementary Information S5}

$\left.{ }^{3} J_{\mathrm{H}-\mathrm{H}}=9 \mathrm{~Hz},{ }^{4} \mathrm{~J}_{\mathrm{F}-\mathrm{H}}=18 \mathrm{~Hz}, 3-\mathrm{Ar}\right), 3.91\left(2 \mathrm{H}, \mathrm{s}, \mathrm{BrCH}_{2}\right) ;{ }^{13} \mathrm{C}$ $\operatorname{NMR}\left(\mathrm{CD}_{3} \mathrm{CN}\right) \delta=165.1(\mathrm{C}=\mathrm{O}), 158.7(\mathrm{~s}, 1-\mathrm{Ph}), 147.7\left(\mathrm{~d},{ }^{1} J_{\mathrm{F}-}\right.$ $\mathrm{C}=2600 \mathrm{~Hz}, 4-\mathrm{Ph}), 122.0\left(\mathrm{~d},{ }^{3} J_{\mathrm{F}-\mathrm{C}}=8 \mathrm{~Hz}, 2-\mathrm{Ph}\right), 115.5\left(\mathrm{~d},{ }^{2} J_{\mathrm{F}-\mathrm{C}}\right.$ $=20 \mathrm{~Hz}, 3-\mathrm{Ph}), 29.8\left(\mathrm{BrCH}_{2}\right) ; \mathrm{m} / \mathrm{z}(\mathrm{MALDI}+) 234(100 \%$, $\left.[\mathrm{M}+\mathrm{H}]^{+}\right), 256\left(45 \%,[\mathrm{M}+\mathrm{Na}]^{+}\right)$, an appropriate isotope pattern was observed.

Phenyl bromoacetamide (5). The title compound was obtained as colorless crystals $(4.0 \mathrm{~g}, 76 \%) . \mathrm{Mp}=125-128.5$ ${ }^{\circ} \mathrm{C} ;{ }^{1} \mathrm{H}$ NMR $\left(400 \mathrm{MHz}, \mathrm{CD}_{3} \mathrm{CN}\right) \delta=10.25(1 \mathrm{H}, \mathrm{s}$ br, NH), $7.41\left(2 \mathrm{H}, \mathrm{d},{ }^{3} J_{\mathrm{H}-\mathrm{H}}=8 \mathrm{~Hz}, \mathrm{Ar}\right), 7.07\left(2 \mathrm{H}, \mathrm{d},{ }^{3} J_{\mathrm{H}-\mathrm{H}}=8 \mathrm{~Hz}, \mathrm{Ar}\right)$, $3.97\left(2 \mathrm{H}, \mathrm{s}, \mathrm{BrCH}_{2}\right) ;{ }^{13} \mathrm{C}$ NMR $\left(\mathrm{CD}_{3} \mathrm{CN}\right) \delta=164.3(\mathrm{C}=\mathrm{O})$, 135.9 (1-Ar), 132.6 (4-Ar), 129.0 (2-Ar, 3-Ar), $30.3\left(\mathrm{BrCH}_{2}\right)$; $\mathrm{m} / \mathrm{z}(\mathrm{MALDI}+) 216\left(100 \%,[\mathrm{M}+\mathrm{H}]^{+}\right)$, an appropriate isotope pattern was observed; Anal. Found C $44.4 \%$, H $3.8 \%$, N 6.3 $\%, \mathrm{C}_{8} \mathrm{H}_{9} \mathrm{BrNO}$ requires $\mathrm{C} 44.6 \%, \mathrm{H} 4.1 \%, \mathrm{~N} 6.5 \%$.

4-Methyoxyphenyl bromoacetamide (6). The title compound was obtained as colorless solid (4.8 g, $81 \%) . \quad \mathrm{Mp}=128-$ $129.5{ }^{\circ} \mathrm{C} ;{ }^{1} \mathrm{H}$ NMR $\left(400 \mathrm{MHz}, \mathrm{CD}_{3} \mathrm{CN}\right) \delta=8.64(1 \mathrm{H}, \mathrm{s} \mathrm{br}$, $\mathrm{NH}), 7.46\left(2 \mathrm{H}, \mathrm{d},{ }^{3} J_{\mathrm{H}-\mathrm{H}}=9 \mathrm{~Hz}, \mathrm{Ar}\right), 6.91\left(2 \mathrm{H}, \mathrm{d},{ }^{3} \mathrm{~J}_{\mathrm{H}-\mathrm{H}}=9 \mathrm{~Hz}\right.$, $\mathrm{Ar}), 3.94\left(2 \mathrm{H}, \mathrm{s}, \mathrm{BrCH}_{2}\right), 3.77\left(3 \mathrm{H}, \mathrm{s}, \mathrm{OCH}_{3}\right) ;{ }^{13} \mathrm{C} \mathrm{NMR}$ $\left(\mathrm{CD}_{3} \mathrm{CN}\right) \delta=164.8(\mathrm{C}=\mathrm{O}), 156.8(4-\mathrm{Ar}), 131.5(1-\mathrm{Ar}), 121.9$ (3-Ar), $114.2(2-\mathrm{Ar}), \quad 55.3 \quad\left(\mathrm{OCH}_{3}\right), 29.9 \quad\left(\mathrm{BrCH}_{2}\right) ; \mathrm{m} / \mathrm{z}$ $(\mathrm{MALDI}+) 246\left(100 \%,[\mathrm{M}+\mathrm{H}]^{+}\right), 267\left(85 \%,[\mathrm{M}+\mathrm{Na}]^{+}\right)$, an appropriate isotope pattern was observed; Anal. Found C 44.0 $\%, \mathrm{H} 4.1 \%, \mathrm{~N} 5.4 \%, \mathrm{C}_{9} \mathrm{H}_{11} \mathrm{BrNO}_{2}$ requires C $44.1 \%, \mathrm{H} 4.5 \%$, N $5.7 \%$.

4-Cyanophenyl bromoacetamide (7). The title compound was obtained as white solid $(0.90 \mathrm{~g}, 81 \%)$. Mp, sublimes above $\sim 155{ }^{\circ} \mathrm{C} ;{ }^{1} \mathrm{H}$ NMR $\left(400 \mathrm{MHz}, \mathrm{CD}_{3} \mathrm{CN}\right) \delta=9.84(1 \mathrm{H}, \mathrm{s}$ br, $\mathrm{NH}), 7.78\left(2 \mathrm{H}, \mathrm{d},{ }^{3} J_{\mathrm{H}-\mathrm{H}}=8 \mathrm{~Hz}, \mathrm{Ar}\right), 7.65\left(2 \mathrm{H}, \mathrm{d},{ }^{3} J_{\mathrm{H}-\mathrm{H}}=8 \mathrm{~Hz}\right.$, Ar), $4.00\left(2 \mathrm{H}, \mathrm{s}, \mathrm{BrCH}_{2}\right) ;{ }^{13} \mathrm{C}$ NMR $\left(100 \mathrm{MHz}, \mathrm{CD}_{3} \mathrm{CN}\right) \delta=$ $165.5(\mathrm{C}=\mathrm{O}), 142.9$ (Ar), 133.3 (2-Ar, 3-Ar) $117.4(\mathrm{CN}), 107.0$ (Ar), $\left.29.5\left(\mathrm{BrCH}_{2}\right) ; \mathrm{m} / \mathrm{z}(\mathrm{MALDI}+) 241(100 \% \text {, [M+H] }]^{+}\right)$, an appropriate isotope pattern was observed; Anal. Found C 44.9 $\%, \mathrm{H} 2.8 \%, \mathrm{~N} 11.4 \%, \mathrm{C}_{9} \mathrm{H}_{7} \mathrm{BrN}_{2} \mathrm{O}$ requires C $45.2 \%, \mathrm{H} 2.9$ $\%$, N $11.7 \%$.

4-tert-Butyl (2-bromoacetamido)benzoate (8). The title compound was obtained as off white solid $(1.5 \mathrm{~g}, 60 \%)$. Mp = $111-113{ }^{\circ} \mathrm{C}$; ${ }^{1} \mathrm{H}$ NMR $\left(400 \mathrm{MHz}, \mathrm{CDCl}_{3}\right) \delta=8.54(1 \mathrm{H}, \mathrm{s}$ br, $\mathrm{NH}), 7.90\left(2 \mathrm{H}, \mathrm{d},{ }^{3} J_{\mathrm{H}-\mathrm{H}}=8 \mathrm{~Hz}, \mathrm{Ar}\right), 7.56\left(2 \mathrm{H}, \mathrm{d},{ }^{3} J_{\mathrm{H}-\mathrm{H}}=8 \mathrm{~Hz}\right.$, $\mathrm{Ar}), 4.13\left(2 \mathrm{H}, \mathrm{s}, \mathrm{BrCH}_{2}\right), 1.52\left(9 \mathrm{H}, \mathrm{s}, \mathrm{C}\left(\mathrm{CH}_{3}\right)_{3}\right) ;{ }^{13} \mathrm{C} \mathrm{NMR}$ $\left(\mathrm{CDCl}_{3}\right) \delta 165.3(\mathrm{C}=\mathrm{O}), 164.3(\mathrm{C}=\mathrm{O}), 140.6(\mathrm{Ar}), 130.7(\mathrm{Ar})$, 128.4 (Ar), $119.2(\mathrm{Ar}), 81.2\left(\underline{\mathrm{C}}\left(\mathrm{CH}_{3}\right)_{3}\right) 43.0\left(\mathrm{BrCH}_{2}\right), 28.3$ $\left(\mathrm{C}\left(\underline{\mathrm{CH}}_{3}\right)_{3}\right) ; \quad \mathrm{m} / \mathrm{z} \quad(\mathrm{MALDI}+) \quad 316 \quad\left(100 \%, \quad[\mathrm{M}+\mathrm{H}]^{+}\right), \quad$ an appropriate isotope pattern was observed; Anal. Found C 57.4 $\%, \mathrm{H} 6.0 \%, \mathrm{~N} 5.0 \%, \mathrm{C}_{13} \mathrm{H}_{17} \mathrm{ClNO}_{3}$ requires C $57.9 \%, \mathrm{H} 6.3$ $\%$, N $5.2 \%$.

4-Nitrophenyl bromoacetamide (9). The title compound was obtained as yellow crystals $(3.3 \mathrm{~g}, 64 \%) . \mathrm{Mp}=174-179{ }^{\circ} \mathrm{C}$; ${ }^{1} \mathrm{H}$ NMR $\left(400 \mathrm{MHz}, \mathrm{CD}_{3} \mathrm{CN}\right) \delta=9.20(1 \mathrm{H}, \mathrm{s}$ br, NH), 8.27, $\left(2 \mathrm{H}, \mathrm{d},{ }^{3} J_{\mathrm{H}-\mathrm{H}}=8 \mathrm{~Hz}, \mathrm{Ar}\right), 7.80\left(2 \mathrm{H}, \mathrm{d},{ }^{3} J_{\mathrm{H}-\mathrm{H}}=8 \mathrm{~Hz}, \mathrm{Ar}\right), 4.03$ $\left(2 \mathrm{H}, \mathrm{s}, \mathrm{BrCH}_{2}\right) ;{ }^{13} \mathrm{C} \mathrm{NMR}\left(\mathrm{CD}_{3} \mathrm{CN}\right) \delta=165.8(\mathrm{C}=\mathrm{O}), 144.5$ (Ar), 143.8 (Ar), $125.1(\mathrm{Ar}), 119.4(\mathrm{Ar}), 29.6\left(\mathrm{BrCH}_{2}\right) ; \mathrm{m} / \mathrm{z}$ (MALDI+) $261\left(100 \%,[\mathrm{M}+\mathrm{H}]^{+}\right)$, an appropriate isotope pattern was observed; Anal. Found C $37.0 \%$, H $2.6 \%$, N 10.6 $\%, \mathrm{C}_{8} \mathrm{H}_{7} \mathrm{BrN}_{2} \mathrm{O}_{3}$ requires $\mathrm{C} 37.1 \%, \mathrm{H} 2.7 \%$, N $10.8 \%$.
General procedure for the preparation of ligands. Potassium carbonate (46 mg, $0.33 \mathrm{mmol}$ ) was added to a solution of the triacetamide $2(0.20 \mathrm{~g}, 0.33 \mathrm{mmol})$ in acetonitrile $(50 \mathrm{~mL})$ and mixture stirred. The $p$ bromoacetamide $6(81 \mathrm{mg}, 033 \mathrm{mmol})$ was added and the reaction heated to $60{ }^{\circ} \mathrm{C}$ for 24 hours with stirring. The reaction was cooled to room temperature and filtered to remove the inorganic salts. The title compound was purified by column chromatography over silica gel eluting with $15 \%$ methanol in dichloromethane.

1- $N$-(4-Methylphenyl)-4,7,10-tris-( $N$-ethylacetate)-1,4,7,10tetraazacyclododecane-1,4,7,10-tetraacetamide (10). The title compound was obtained as a pale yellow solid $(0.14 \mathrm{~g}, 51$ \%). $R_{\mathrm{f}}=0.40\left(\mathrm{SiO}_{2}, 15 \% \mathrm{MeOH}\right.$ in $\left.\mathrm{CH}_{2} \mathrm{Cl}_{2}\right) ;{ }^{1} \mathrm{H}$ NMR $(400$ $\left.\mathrm{MHz}, \mathrm{CD}_{3} \mathrm{CN}\right) \delta=7.59\left(2 \mathrm{H}, \mathrm{d},{ }^{3} J_{\mathrm{H}-\mathrm{H}}=10 \mathrm{~Hz}, \mathrm{Ar}\right), 7.15(2 \mathrm{H}, \mathrm{d}$, $\left.{ }^{3} J_{\mathrm{H}-\mathrm{H}}=10 \mathrm{~Hz}, \mathrm{Ar}\right), 4.14\left(6 \mathrm{H}, \mathrm{m}, 2 \times \mathrm{OCH}_{2}\right), 3.4-3.8(14 \mathrm{H}, \mathrm{m}$ br, $\left.\mathrm{NCH}_{2} \mathrm{CO}, \mathrm{NHCH}_{2} \mathrm{CO}_{2} \mathrm{Et}\right), 2.7-2.9\left(16 \mathrm{H}, \mathrm{m}\right.$ br, ring $\left.\mathrm{CH}_{2} \mathrm{~N}\right)$, $2.3\left(3 \mathrm{H}, \mathrm{s}, \mathrm{CH}_{3}\right), 1.26\left(6 \mathrm{H}, \mathrm{t},{ }^{3} J_{\mathrm{H}-\mathrm{H}}=8 \mathrm{~Hz}, \mathrm{CH}_{3}\right), 1.23(3 \mathrm{H}, \mathrm{t}$, $\left.{ }^{3} J_{\mathrm{H}-\mathrm{H}}=8 \mathrm{~Hz}, \mathrm{CH}_{3}\right) ;{ }^{13} \mathrm{C} \mathrm{NMR}\left(100 \mathrm{MHz}, \mathrm{CD}_{3} \mathrm{CN}\right) \delta=172.6$ $(\mathrm{C}=\mathrm{O}), 171.7(2 \times \mathrm{C}=\mathrm{O}), 170.6(\mathrm{C}=\mathrm{O}), 170.0(\mathrm{C}=\mathrm{O}), 136.3(1-$ Ar), 129.5 (3-Ar), 129.3 (4-Ar), 120.3 (3-Ar), $61.2\left(2 \times \mathrm{OCH}_{2}\right)$, 57.5 (br, ring $\mathrm{CH}_{2} \mathrm{~N}$ ), 52.2 (br, $\mathrm{NCH}_{2} \mathrm{CO}$ ), 50.2 (ring $\mathrm{CH}_{2} \mathrm{~N}$ ), $41.3\left(\mathrm{NHCH}_{2} \mathrm{CO}_{2} \mathrm{Et}\right), 41.0\left(\mathrm{NHCH}_{2} \mathrm{CO}_{2} \mathrm{Et}\right), 20.1\left(\mathrm{CH}_{3}\right), 13.7(2$ $\left.\times \mathrm{CH}_{3}\right) ; \mathrm{m} / \mathrm{z}(\mathrm{MALDI}+) 749(100 \%)[\mathrm{M}+\mathrm{H}]^{+}, 787(85 \%$ $\left.[\mathrm{M}+\mathrm{K}]^{+}\right)$; Anal. found $\mathrm{C} 46.9 \%, \mathrm{H} 6.6 \%$, N $11.6 \%$, $\mathrm{C}_{35} \mathrm{H}_{56} \mathrm{~N}_{8} \mathrm{O}_{10} \cdot \mathrm{KHCO}_{3} \cdot 4.4 \mathrm{H}_{2} \mathrm{O}$ requires $\mathrm{C} 46.6 \%$, H $7.1 \%$, N $12.1 \%$.

1- $N$-(4-Fluorophenyl)-4,7,10-tris-( $N$-ethylacetate)-1,4,7,10tetraazacyclododecane-1,4,7,10-tetraacetamide (11). The title compound was obtained as a colourless solid. (0.19 g, 66 \%). $R_{\mathrm{f}}=0.45\left(\mathrm{SiO}_{2}, 15 \% \mathrm{MeOH}\right.$ in $\left.\mathrm{CH}_{2} \mathrm{Cl}_{2}\right) ; \mathrm{Mp}=116-119$ ${ }^{\circ} \mathrm{C}$; ${ }^{1} \mathrm{H}$ NMR $\left(400 \mathrm{MHz}, \mathrm{CD}_{3} \mathrm{CN}\right) \delta=7.73(2 \mathrm{H}$, br, Ar), 7.06 $(2 \mathrm{H}, \mathrm{br}, \mathrm{Ar}), 4.14\left(6 \mathrm{H}, \mathrm{m}, 2 \times \mathrm{OCH}_{2}\right), 3.3-3.8(14 \mathrm{H}, \mathrm{m}$ br, $\left.\mathrm{NCH}_{2} \mathrm{CO}, \mathrm{NHCH}_{2} \mathrm{CO}_{2} \mathrm{Et}\right), 2.4-2.6\left(16 \mathrm{H}, \mathrm{m}\right.$ br, ring $\left.\mathrm{CH}_{2} \mathrm{~N}\right)$, $1.25\left(6 \mathrm{H}, \mathrm{t},{ }^{3} J_{\mathrm{H}-\mathrm{H}}=8 \mathrm{~Hz}, \mathrm{CH}_{3}\right), 1.23\left(3 \mathrm{H}, \mathrm{t},{ }^{3} J_{\mathrm{H}-\mathrm{H}}=8 \mathrm{~Hz}, \mathrm{CH}_{3}\right)$; ${ }^{13} \mathrm{C}$ NMR $\left(100 \mathrm{MHz}, \mathrm{CD}_{3} \mathrm{CN}\right) \delta=173.2(\mathrm{C}=\mathrm{O}), 172.8(2 \times$ $\mathrm{C}=\mathrm{O}), 170.0(\mathrm{C}=\mathrm{O}), 169.9(\mathrm{C}=\mathrm{O}), 157.4$ (s, 1-Ar), 147.9 (d, $\left.{ }^{1} J_{\mathrm{C}-\mathrm{F}}=2490 \mathrm{~Hz}, 4-\mathrm{Ar}\right), 121.9\left(\mathrm{~d},{ }^{2} J_{\mathrm{C}-\mathrm{F}}=41 \mathrm{~Hz}, 3-\mathrm{Ar}\right), 115.3(\mathrm{~d}$, $\left.{ }^{3} J_{\mathrm{C}-\mathrm{F}}=22 \mathrm{~Hz}, 2-\mathrm{Ar}\right), 61.2\left(2 \times \mathrm{OCH}_{2}\right), 57.4\left(\mathrm{br}, \mathrm{NCH}_{2} \mathrm{CO}\right)$, 56.9 (br, $\left.\mathrm{NCH}_{2} \mathrm{CO}\right), 53.6\left(\mathrm{NCH}_{2} \mathrm{CO}\right), 50.7$ (ring $\left.\mathrm{CH}_{2} \mathrm{~N}\right), 41.3$ $\left(\mathrm{NHCH}_{2} \mathrm{CO}_{2} \mathrm{Et}\right), 41.1\left(\mathrm{NHCH}_{2} \mathrm{CO}_{2} \mathrm{Et}\right), 13.6\left(2 \times \mathrm{CH}_{3}\right) ; \mathrm{m} / \mathrm{z}$ (MALDI+) $753\left(100 \%,[\mathrm{M}+\mathrm{H}]^{+}\right)$; Anal. found C $45.3 \%, \mathrm{H} 6.3$ $\%, \mathrm{~N} 11.6 \%, \mathrm{C}_{34} \mathrm{H}_{53} \mathrm{FN}_{8} \mathrm{O}_{10} \cdot \mathrm{KHCO}_{3} \cdot 4.4 \mathrm{H}_{2} \mathrm{O}$ requires $\mathrm{C} 45.1$ $\%$, H $6.8 \%$, N $12.0 \%$.

1- $N$-Phenyl-4,7,10-tris-( $N$-ethylacetate)-1,4,7,10-tetraazacyclododecane-1,4,7,10-tetraacetamide (12). The title compound was obtained as a colourless amorphous solid (0.15 $\mathrm{mg}, 52 \%) . R_{\mathrm{f}}=0.35\left(\mathrm{SiO}_{2}, 20 \% \mathrm{MeOH}\right.$ in $\left.\mathrm{CH}_{2} \mathrm{Cl}_{2}\right) ; \mathrm{Mp}=32-$ $34{ }^{\circ} \mathrm{C}$; ${ }^{1} \mathrm{H}$ NMR $\left(400 \mathrm{MHz}, \mathrm{CD}_{3} \mathrm{CN}\right) \delta=7.47$ ( $\left.2 \mathrm{H}, \mathrm{m}, \mathrm{Ar}\right)$, $7.17(2 \mathrm{H}, \mathrm{m}, \mathrm{Ar}), 6.93(1 \mathrm{H}, \mathrm{m}, 4-\mathrm{Ar}), 3.94\left(6 \mathrm{H}, \mathrm{m}, \mathrm{OCH}_{2}\right), 3.70$ $\left(6 \mathrm{H}, \mathrm{m}, \mathrm{NHC}_{2} \mathrm{CO}_{2} \mathrm{Et}\right), 3.16\left(8 \mathrm{H}, \mathrm{m}, \mathrm{NCH}_{2} \mathrm{CO}\right), 2.2-2.7$ $\left(16 \mathrm{H}, \mathrm{m}\right.$ br, ring $\left.\mathrm{CH}_{2} \mathrm{~N}\right), 1.06\left(9 \mathrm{H}, \mathrm{m}, \mathrm{CH}_{3}\right) ;{ }^{13} \mathrm{C}$ NMR $(100$ $\left.\mathrm{MHz}, \mathrm{CD}_{3} \mathrm{CN}\right) \delta=172.5(2 \times \mathrm{C}=\mathrm{O}), 170.5(\mathrm{C}=\mathrm{O}), 170.1$ $(\mathrm{C}=\mathrm{O}), 170.0(\mathrm{C}=\mathrm{O}), 142.6$ (Ar), 129.2 (Ar), 124.0 (Ar), 117.6 (Ar), $61.2\left(2 \times \mathrm{OCH}_{2}\right), 58.3$ (br ring $\left.\mathrm{CH}_{2} \mathrm{~N}\right), 53.1$ (br ring $\left.\mathrm{CH}_{2} \mathrm{~N}\right), 46.8\left(\mathrm{NCH}_{2} \mathrm{CO}\right), 46.4\left(\mathrm{NCH}_{2} \mathrm{CO}\right), 44.8\left(\mathrm{NCH}_{2} \mathrm{CO}\right)$, $41.0\left(\mathrm{NHCH}_{2} \mathrm{CO}_{2}\right), 13.7\left(2 \times \mathrm{CH}_{3}\right) . \mathrm{m} / \mathrm{z}(\mathrm{MALDI}+) 735(100 \%$, $\left.[\mathrm{M}+\mathrm{H}]^{+}\right)$. 


\section{ja076325y: Ratnakar et al. Supplementary Information S6}

1- $N$-(4-Methoxyphenyl)-4,7,10-tris-( $N$-ethylacetate)-1,4,7, 10-tetraazacyclododecane-1,4,7,10-tetraacetamide (13). The title compound was obtained as a colourless solid $(0.18 \mathrm{~g}, 64$ \%). $R_{\mathrm{f}}=0.40\left(\mathrm{SiO}_{2}, 20 \% \mathrm{MeOH}\right.$ in $\left.\mathrm{CH}_{2} \mathrm{Cl}_{2}\right) ; \mathrm{Mp}=137.5-139$ ${ }^{\circ} \mathrm{C}$; ${ }^{1} \mathrm{H}$ NMR $\left(400 \mathrm{MHz}, \mathrm{CD}_{3} \mathrm{CN}\right) \delta=7.62\left(2 \mathrm{H}, \mathrm{d},{ }^{3} J_{\mathrm{H}-\mathrm{H}}=10\right.$ $\mathrm{Hz}, \mathrm{Ar}), 6.86\left(2 \mathrm{H}, \mathrm{d},{ }^{3} \mathrm{~J}_{\mathrm{H}-\mathrm{H}}=10 \mathrm{~Hz}, \mathrm{Ar}\right), 4.11(6 \mathrm{H}, \mathrm{m}, 2 \times$ $\left.\mathrm{OCH}_{2}\right), 3.74\left(3 \mathrm{H}, \mathrm{s}, \mathrm{OCH}_{3}\right), 3.2-3.9\left(14 \mathrm{H}, \mathrm{m}\right.$ br, $\mathrm{NCH}_{2} \mathrm{CO}$, $\left.\mathrm{NHCH}_{2} \mathrm{CO}_{2} \mathrm{Et}\right), 2.2-2.8\left(16 \mathrm{H}, \mathrm{m}\right.$ br, ring $\left.\mathrm{CH}_{2} \mathrm{~N}\right), 1.22(6 \mathrm{H}, \mathrm{t}$, $\left.{ }^{3} J_{\mathrm{H}-\mathrm{H}}=8 \mathrm{~Hz}, \mathrm{CH}_{3}\right), 1.21\left(3 \mathrm{H}, \mathrm{t},{ }^{3} J_{\mathrm{H}-\mathrm{H}}=8 \mathrm{~Hz}, \mathrm{CH}_{3}\right) ;{ }^{13} \mathrm{C} \mathrm{NMR}$ $\left(100 \mathrm{MHz}, \mathrm{CD}_{3} \mathrm{CN}\right) \delta=173.2(\mathrm{C}=\mathrm{O}), 172.8(2 \times \mathrm{C}=\mathrm{O}), 170.0$ $(2 \times \mathrm{C}=\mathrm{O}), 156.3$ (4-Ar), 132.1 (1-Ar), 121.9 (3-Ar), 114.0 (2$\mathrm{Ar}), 61.2\left(2 \times \mathrm{OCH}_{2}\right), 57.5\left(\mathrm{br}\right.$, ring $\left.\mathrm{CH}_{2} \mathrm{~N}\right) 55.4\left(\mathrm{OCH}_{3}\right), 53.2$ $\left(\mathrm{NCH}_{2} \mathrm{CO}\right), 52.8 \quad\left(\mathrm{NCH}_{2} \mathrm{CO}\right), 52.2 \quad\left(\mathrm{NCH}_{2} \mathrm{CO}\right), 50.7$ (ring $\left.\mathrm{CH}_{2} \mathrm{~N}\right), 41.2\left(\mathrm{NHCH}_{2} \mathrm{CO}_{2} \mathrm{Et}\right), 41.1\left(\mathrm{NHCH}_{2} \mathrm{CO}_{2} \mathrm{Et}\right), 13.3(2 \times$ $\left.\mathrm{CH}_{3}\right) ; \quad \mathrm{m} / \mathrm{z} \quad$ (MALDI+) $765 \quad(70 \%) \quad[\mathrm{M}+\mathrm{H}]^{+}, \quad 787 \quad(70 \%$, $\left.[\mathrm{M}+\mathrm{Na}]^{+}\right), 805\left(100 \%,[\mathrm{M}+\mathrm{K}]^{+}\right)$, Anal. found $\mathrm{C} 46.5 \%, \mathrm{H} 6.1$ $\%, \mathrm{~N} 13.0 \%, \mathrm{C}_{35} \mathrm{H}_{56} \mathrm{~N}_{8} \mathrm{O}_{11} \cdot 0.9 \mathrm{KBr} \cdot \mathrm{H}_{2} \mathrm{O}$ requires $\mathrm{C} 47.0 \%, \mathrm{H}$ $6.5 \%, \mathrm{~N} 12.5 \%$.

1- $N$-(4-Cyanophenyl)-4,7,10-tris-( $N$-ethylacetate)-1,4,7,10tetraazacyclododecane-1,4,7,10-tetraacetamide (14). The title compound was obtained as an off-white solid (0.16 g, 55 \%). $R_{\mathrm{f}}=0.54\left(\mathrm{SiO}_{2}, 15 \% \mathrm{MeOH}\right.$ in $\left.\mathrm{CH}_{2} \mathrm{Cl}_{2}\right) ; \mathrm{Mp}=94.5-96$ ${ }^{\circ} \mathrm{C} ;{ }^{1} \mathrm{H}$ NMR $\left(400 \mathrm{~Hz}, \mathrm{CD}_{3} \mathrm{CN}\right) \delta=7.86\left(2 \mathrm{H}, \mathrm{d},{ }^{3} J_{\mathrm{H}-\mathrm{H}}=8 \mathrm{~Hz}\right.$, Ar), $7.69\left(2 \mathrm{H}, \mathrm{d},{ }^{3} J_{\mathrm{H}-\mathrm{H}}=8 \mathrm{~Hz}, \operatorname{Ar}\right), 4.15\left(4 \mathrm{H}, \mathrm{q},{ }^{3} J_{\mathrm{H}-\mathrm{H}}=7 \mathrm{~Hz}\right.$, $\left.\mathrm{OCH}_{2}\right), 4.14\left(2 \mathrm{H}, \mathrm{q},{ }^{3} J_{\mathrm{H}-\mathrm{H}}=7 \mathrm{~Hz}, \mathrm{OCH}_{2}\right), 3.87(8 \mathrm{H}, \mathrm{br}$, $\left.\mathrm{NCH}_{2} \mathrm{CO}\right), 2.92\left(6 \mathrm{H}, \mathrm{m}\right.$ br, $\left.\mathrm{NHCH}_{2} \mathrm{CO}_{2} \mathrm{Et}\right), 2.29(16 \mathrm{H}, \mathrm{s}$ br, ring $\left.\mathrm{CH}_{2} \mathrm{~N}\right), 1.24\left(9 \mathrm{H}, \mathrm{t},{ }^{3} \mathrm{~J}_{\mathrm{H}-\mathrm{H}}=7 \mathrm{~Hz}, 2 \times \mathrm{CH}_{3}\right) ;{ }^{13} \mathrm{C} \mathrm{NMR}(100$ $\left.\mathrm{MHz}, \mathrm{CD}_{3} \mathrm{CN}\right) \delta=172.7(\mathrm{C}=\mathrm{O}), 172.3(2 \times \mathrm{C}=\mathrm{O}), 170.1$ $(\mathrm{C}=\mathrm{O}), 166.3(\mathrm{C}=\mathrm{O}), 143.3$ (4-Ar), 142.6 (1-Ar), 133.4 (3-Ar), 119.8 (2-Ar), 118. $5(\mathrm{CN}), 62.3\left(\mathrm{NCH}_{2} \mathrm{CO}\right), 61.3\left(2 \times \mathrm{OCH}_{2}\right)$, $58.3\left(\mathrm{NCH}_{2} \mathrm{CO}\right), 57.1\left(\mathrm{NCH}_{2} \mathrm{CO}\right), 51.0\left(\right.$ ring $\left.\mathrm{CH}_{2} \mathrm{~N}\right), 41.2$ $\left(\mathrm{NHCH}_{2} \mathrm{CO}_{2} \mathrm{Et}\right), 41.1\left(\mathrm{NH}_{\underline{C}} \mathrm{H}_{2} \mathrm{CO}_{2} \mathrm{Et}\right), 13.9\left(2 \times \mathrm{CH}_{3}\right) ; \mathrm{m} / \mathrm{z}$ $(\mathrm{MALDI}+) 760\left(80 \%,[\mathrm{M}+\mathrm{H}]^{+}\right), 782\left(100 \%,[\mathrm{M}+\mathrm{Na}]^{+}\right), 798$ $\left(10 \%,[\mathrm{M}+\mathrm{K}]^{+}\right)$; Anal. found $\mathrm{C} 46.7 \%$, H $6.3 \%$, N $13.3 \%$, $\mathrm{C}_{35} \mathrm{H}_{53} \mathrm{~N}_{9} \mathrm{O}_{10} \cdot 0.9 \mathrm{KBr} \cdot 2.2 \mathrm{H}_{2} \mathrm{O}$ requires $\mathrm{C} 46.2 \%, \mathrm{H} 6.4 \%, \mathrm{~N}$ $13.8 \%$.

1- $N$-(4-(tert-Butoxycarbonyl)phenyl)-4,7,10-tris-( $N$-ethylacetate)-1,4,7,10-tetraazacyclododecane-1,4,7,10-tetraacetamide (15). The title compound was obtained as a colourless solid $(0.17 \mathrm{~g}, 60 \%) . R_{\mathrm{f}}=0.43\left(\mathrm{SiO}_{2}, 15 \% \mathrm{MeOH}\right.$ in $\left.\mathrm{CH}_{2} \mathrm{Cl}_{2}\right)$; ${ }^{1} \mathrm{H}$ NMR $\left(400 \mathrm{MHz}, \mathrm{CD}_{3} \mathrm{CN}\right) \delta=8.29\left(2 \mathrm{H}, \mathrm{d},{ }^{3} J_{\mathrm{H}-\mathrm{H}}=8 \mathrm{~Hz}\right.$, Ar), $8.14\left(2 \mathrm{H}, \mathrm{d},{ }^{3} J_{\mathrm{H}-\mathrm{H}}=8 \mathrm{~Hz}, \mathrm{Ar}\right), 4.66(6 \mathrm{H}, \mathrm{m}$, $\left.\mathrm{NHCH}_{2} \mathrm{CO}_{2} \mathrm{Et}\right), 4.49\left(6 \mathrm{H}, \mathrm{m}, \mathrm{OCH}_{2}\right) 4.25(8 \mathrm{H}, \mathrm{m}$ br, $\left.\mathrm{NCH}_{2} \mathrm{CO}\right), 3.45\left(8 \mathrm{H}\right.$, br, ring $\left.\mathrm{CH}_{2} \mathrm{~N}\right), 3.03\left(8 \mathrm{H}\right.$, br, ring $\left.\mathrm{CH}_{2} \mathrm{~N}\right)$, $1.94\left(9 \mathrm{H}, \mathrm{s}, \mathrm{C}\left(\mathrm{CH}_{3}\right)_{3}\right), 1.59\left(9 \mathrm{H}, \mathrm{t},{ }^{3} \mathrm{~J}_{\mathrm{H}-\mathrm{H}}=8 \mathrm{~Hz}, 2 \times \mathrm{CH}_{3}\right) ;{ }^{13} \mathrm{C}$ NMR $\left(100 \mathrm{MHz}, \mathrm{CD}_{3} \mathrm{CN}\right) \delta=173.4(2 \times \mathrm{C}=\mathrm{O}), 173.0(2 \times$ $\mathrm{C}=\mathrm{O}), 170.8(\mathrm{C}=\mathrm{O}), 166.3(\mathrm{C}=\mathrm{O}), 143.4(4-\mathrm{Ar}), 131.2(3-\mathrm{Ar})$, 128.4 (2-Ar), 118.4 (1-Ar), $81.6\left(\underline{\mathrm{C}}\left(\mathrm{CH}_{3}\right)_{3}\right), 61.1\left(2 \times \mathrm{OCH}_{2}\right)$, 59.2 (br, $\left.2 \times \mathrm{NCH}_{2} \mathrm{CO}\right), 57.8\left(\mathrm{NCH}_{2} \mathrm{CO}\right), 53.63\left(\right.$ ring $\left.\mathrm{CH}_{2} \mathrm{~N}\right)$, 51.28 (ring $\left.\mathrm{CH}_{2} \mathrm{~N}\right), 44,5\left(\mathrm{NHCH}_{2} \mathrm{CO}_{2} \mathrm{Et}\right), 41.9\left(\mathrm{NHCH}_{2} \mathrm{CO}_{2} \mathrm{Et}\right)$, $28.4\left(\mathrm{C}\left(\underline{\mathrm{CH}}_{3}\right)_{3}\right), 14.6\left(2 \times \mathrm{CH}_{3}\right) ; \mathrm{m} / \mathrm{z}(\mathrm{MALDI}+) 835(100 \%$, $\left.[\mathrm{M}+\mathrm{H}]^{+}\right), 857\left(20 \%,[\mathrm{M}+\mathrm{Na}]^{+}\right)$.

1- $N$-(4-Nitrophenyl)-4,7,10-tris-( $N$-ethylacetate)-1,4,7,10tetraazacyclododecane-1,4,7,10-tetraacetamide (16). The title compound was obtained as a yellow solid (0.18 g, $64 \%)$. $R_{\mathrm{f}}=0.48\left(\mathrm{SiO}_{2}, 10 \% \mathrm{MeOH}\right.$ in $\left.\mathrm{CH}_{2} \mathrm{Cl}_{2}\right) ; \mathrm{Mp}=92-94{ }^{\circ} \mathrm{C} ;{ }^{1} \mathrm{H}$ NMR (400 MHz, CD $\left.\mathrm{CD}_{3} \mathrm{CN}\right) \delta=8.18\left(2 \mathrm{H}, \mathrm{d},{ }^{3} J_{\mathrm{H}-\mathrm{H}}=8 \mathrm{~Hz}, \mathrm{Ar}\right)$, $7.40\left(2 \mathrm{H}, \mathrm{d},{ }^{3} J_{\mathrm{H}-\mathrm{H}}=8 \mathrm{~Hz}, \mathrm{Ar}\right), 4.14\left(6 \mathrm{H}, \mathrm{q},{ }^{3} J_{\mathrm{H}-\mathrm{H}}=8 \mathrm{~Hz}, \mathrm{OCH}_{2^{-}}\right.$ overlapping), 3.87 (6H, s br, $\left.\mathrm{NHCH}_{2} \mathrm{CO}_{2} \mathrm{Et}\right), 2.2-3.4(24 \mathrm{H}, \mathrm{m}$ br, ring $\left.\mathrm{CH}_{2} \mathrm{~N}, \mathrm{NCH}_{2} \mathrm{CO}\right), 1.23\left(6 \mathrm{H}, \mathrm{t},{ }^{3} J_{\mathrm{H}-\mathrm{H}}=8 \mathrm{~Hz}, \mathrm{CH}_{3}\right), 1.22$ $\left(3 \mathrm{H}, \mathrm{t},{ }^{3} \mathrm{~J}_{\mathrm{H}-\mathrm{H}}=8 \mathrm{~Hz}, \mathrm{CH}_{3}\right) ;{ }^{13} \mathrm{C} \mathrm{NMR}\left(100 \mathrm{MHz}, \mathrm{CD}_{3} \mathrm{CN}\right) \delta=$ $172.6(\mathrm{C}=\mathrm{O}), 172.3(\mathrm{C}=\mathrm{O}), 170.1(\mathrm{C}=\mathrm{O}), 125.0(\mathrm{Ar}), 144.6$ (Ar), 135.6 (Ar), 119.4 (Ar), $117.5(\mathrm{Ar} \mathrm{CH}), 61.2\left(2 \times \mathrm{CH}_{2} \mathrm{O}\right)$, 58.2 (br, $3 \times \mathrm{NCH}_{2} \mathrm{CO}$ ), 57.0 (br, $2 \times \mathrm{NHCH}_{2} \mathrm{CO}_{2} \mathrm{Et}$ ), 50.9 (br, ring $\left.\mathrm{CH}_{2} \mathrm{~N}\right), 41.1\left(\mathrm{CH}_{2} \mathrm{~N}\right), 13.8\left(2 \times \mathrm{CH}_{3}\right) ; \mathrm{m} / \mathrm{z}(\mathrm{MALDI}+) 780$ $\left(100 \%,[\mathrm{M}+\mathrm{H}]^{+}\right), 802\left(100 \%,[\mathrm{M}+\mathrm{Na}]^{+}\right), 818\left(30 \%,[\mathrm{M}+\mathrm{K}]^{+}\right)$; Anal. found C $45.3 \%$, H $6.0 \%$, N $13.2 \%, \mathrm{C}_{34} \mathrm{H}_{53} \mathrm{~N}_{9} \mathrm{O}_{12} \cdot \mathrm{KH}$ $\mathrm{CO}_{3} \cdot 2.5 \mathrm{H}_{2} \mathrm{O}$ requires $\mathrm{C} 45.5 \%, \mathrm{H} 6.4 \%$, N $13.6 \%$.

General procedure for the synthesis of $\mathbf{E u}^{3+}$ complexes. To a solution of the ligand $(0.15 \mathrm{mmol})$ in acetonitrile $(20 \mathrm{~mL})$ was added a solution of the europium triflate $(0.1 \mathrm{mmol})$ in acetonitrile $(5 \mathrm{~mL})$. The reaction was stirred at room temperature for 18 hours and the solvents removed under reduced pressure. The residue was dried under high vacuum for 3 hours to afford the complex in quantitative yield.

Europium (III) 1- $N$-(4-methylphenyl)-4,7,10-tris-( $N$-ethylacetate)-1,4,7,10-tetraazacyclododecane-1,4,7,10-tetraacetamide triflate salt (Eu10). The complex was obtained as a colourless solid. ${ }^{1} \mathrm{H}$ NMR $\left(400 \mathrm{MHz}, \mathrm{CD}_{3} \mathrm{CN}\right) \delta=24.8(4 \mathrm{H}$, br, ring $\left.a x^{\mathrm{S}}\right), 7.6(2 \mathrm{H}, \mathrm{Ar}), 7.1(2 \mathrm{H}, \mathrm{Ar}), 3.4\left(6 \mathrm{H}, \mathrm{m}, \mathrm{OCH}_{2}\right), 2.0$ $\left(3 \mathrm{H}, \mathrm{s}, \mathrm{ArCH}_{3}\right), 1.1\left(9 \mathrm{H}, \mathrm{m}, \mathrm{CH}_{3}\right),-0.8\left(4 \mathrm{H}, \mathrm{br}\right.$, ring eq $\left.\mathrm{s}^{\mathrm{s}}\right),-2.3$ $\left(4 \mathrm{H}\right.$, br, ring $\left.a x^{\mathrm{C}}\right),-5.1\left(4 \mathrm{H}\right.$, br, ring $\left.e q^{\mathrm{C}}\right),-8.4(4 \mathrm{H}, \mathrm{br}, a c)$, 12.7 (4H, br, ac); m/z (MALDI+) $\left.898(100 \% \text {, [EuL-2H] }]^{+}\right)$, an appropriate isotope pattern was observed.

Europium (III) 1- $N$-(4-fluorophenyl)-4,7,10-tris-( $N$-ethylacetate)-1,4,7,10-tetraazacyclododecane-1,4,7,10-tetraacetamide triflate salt (Eu11). The complex was obtained as a colourless solid. ${ }^{1} \mathrm{H}$ NMR $\left(400 \mathrm{MHz}, \mathrm{CD}_{3} \mathrm{CN}\right) \delta=24.2(4 \mathrm{H}$, br, ring $\left.a x^{\mathrm{S}}\right), 6.9(2 \mathrm{H}, \mathrm{m}, \mathrm{Ar}), 6.4(2 \mathrm{H}, \mathrm{m}, \mathrm{Ar}), 2.3(6 \mathrm{H}, \mathrm{m}$, $\left.\mathrm{OCH}_{2}\right), 0.1\left(9 \mathrm{H}, \mathrm{m}, \mathrm{CH}_{3}\right),-1.8(4 \mathrm{H}, \mathrm{br}$, ring eq $),-4.5(4 \mathrm{H}, \mathrm{br}$, ring $\left.a x^{\mathrm{C}}\right),-7.4\left(4 \mathrm{H}\right.$, br, ring $\left.e q^{\mathrm{C}}\right),-10.6(4 \mathrm{H}, \mathrm{br}, a c),-12.1(4 \mathrm{H}$, br, ac); m/z (MALDI+) $902\left(100 \%,[\mathrm{EuL}-2 \mathrm{H}]^{+}\right)$an appropriate isotope pattern was observed.

Europium (III) 1 - $N$-phenyl-4,7,10-tris-( $N$-ethylacetate)1,4,7,10-tetraazacyclododecane-1,4,7,10-tetraacetamide triflate salt (Eu12). The complex was obtained as a colourless solid. ${ }^{1} \mathrm{H}$ NMR $\left(400 \mathrm{MHz}, \mathrm{CD}_{3} \mathrm{CN}\right) \delta=24.9\left(4 \mathrm{H}\right.$, br, ring $\left.a x^{\mathrm{S}}\right)$, $7.8(2 \mathrm{H}, \mathrm{Ar}), 7.3(2 \mathrm{H}, \mathrm{Ar}), 3.3\left(6 \mathrm{H}, \mathrm{m}, \mathrm{OCH}_{2}\right), 0.9(9 \mathrm{H}, \mathrm{m}$, $\left.\mathrm{CH}_{3}\right),-0.6\left(4 \mathrm{H}\right.$, br, ring $\left.e q^{\mathrm{S}}\right),-2.2\left(4 \mathrm{H}\right.$, br, ring $\left.a x^{\mathrm{C}}\right),-5.3(4 \mathrm{H}$, br, ring $\left.e q^{\mathrm{C}}\right),-8.2(4 \mathrm{H}, \mathrm{br}, a c),-12.5(4 \mathrm{H}, \mathrm{br}, a c) ; \mathrm{m} / \mathrm{z}$ $(\mathrm{MALDI}+) 884\left(100 \%,\left[\mathrm{EuL}-\mathrm{H}_{2}\right]^{+}\right)$, an appropriate isotope pattern was observed.

Europium (III) $1-N$-(4-methoxyphenyl)-4,7,10-tris-( $N$-ethylacetate)-1,4,7,10-tetraazacyclododecane-1,4,7,10-tetraacetamide triflate salt (Eu13). The complex was obtained as a colourless solid. ${ }^{1} \mathrm{H}$ NMR $\left(400 \mathrm{MHz}, \mathrm{CD}_{3} \mathrm{CN}\right) \delta=24.6(4 \mathrm{H}$, br, ring $\left.a x^{\mathrm{S}}\right), 7.5(2 \mathrm{H}, \mathrm{Ar}), 7.0(2 \mathrm{H}, \mathrm{Ar}), 4.2\left(3 \mathrm{H}, \mathrm{s}, \mathrm{ArOCH}_{3}\right)$, $3.4\left(6 \mathrm{H}, \mathrm{m}, \mathrm{OCH}_{2}\right), 1.2\left(9 \mathrm{H}, \mathrm{m}, \mathrm{CH}_{3}\right),-0.6\left(4 \mathrm{H}, \mathrm{br}\right.$, ring eq $\left.\mathrm{s}^{\mathrm{S}}\right)$, $2.5\left(4 \mathrm{H}, \mathrm{br}\right.$, ring $\left.a x^{\mathrm{C}}\right),-4.9\left(4 \mathrm{H}, \mathrm{br}\right.$, ring $\left.e q^{\mathrm{C}}\right),-8.3(4 \mathrm{H}, \mathrm{br}, a c)$, 12.7 (4H, br, ac); m/z (MALDI+) $\left.916\left(100 \% \text {, [EuL- }{ }_{2}\right]^{+}\right)$, an appropriate isotope pattern was observed. 


\section{ja076325y: Ratnakar et al. Supplementary Information S7}

Europium (III) 1- $N$-(4-cyanophenyl)-4,7,10-tris-( $N$-ethylacetate)-1,4,7,10-tetraazacyclododecane-1,4,7,10-tetraacet-

amide triflate salt (Eu14). The complex was obtained as a colourless solid. ${ }^{1} \mathrm{H}$ NMR $\left(400 \mathrm{MHz}, \mathrm{CD}_{3} \mathrm{CN}\right) \delta=27.6(4 \mathrm{H}$, br, ring $\left.a x^{\mathrm{S}}\right), 10.1(4 \mathrm{H}, \mathrm{m}, \mathrm{Ar}), 5.4\left(6 \mathrm{H}, \mathrm{m}, \mathrm{OCH}_{2}\right), 3.5(9 \mathrm{H}, \mathrm{m}$, $\left.\mathrm{CH}_{3}\right), 1.9\left(4 \mathrm{H}\right.$, br, ring $\left.e q^{\mathrm{S}}\right), 0.0\left(4 \mathrm{H}\right.$, br, ring $\left.a x^{\mathrm{C}}\right),-2.3(4 \mathrm{H}$, br, ring $\left.e q^{\mathrm{C}}\right),-5.9(4 \mathrm{H}, \mathrm{br}, a c),-10.3(4 \mathrm{H}, \mathrm{br}, a c) ; \mathrm{m} / \mathrm{z}$ (MALDI + ) $909\left(100 \%,[\mathrm{EuL}-2 \mathrm{H}]^{+}\right)$, an appropriate isotope pattern was observed.

Europium(III) 1- $\mathrm{N}$-(4-(tert-butoxycarbonyl)phenyl)-4,7,10tris-( $N$-ethylacetate)-1,4,7,10-tetraazacyclododecane-1,4,7, 10-tetraacetamide triflate salt (Eu15). The complex was obtained as a colourless solid. ${ }^{1} \mathrm{H}$ NMR $\left(400 \mathrm{MHz}, \mathrm{CD}_{3} \mathrm{CN}\right) \delta$ $=27.7\left(4 \mathrm{H}\right.$, br, ring $\left.a x^{\mathrm{S}}\right), 10.7(2 \mathrm{H}, \mathrm{Ar}), 10.4(2 \mathrm{H}, \mathrm{Ar}), 6.0(6 \mathrm{H}$, $\left.\mathrm{m}, \mathrm{OCH}_{2}\right), 4.6\left(9 \mathrm{H}, \mathrm{m}, \mathrm{CH}_{3}\right), 4.0\left(9 \mathrm{H}, \mathrm{s}, \mathrm{C}\left(\mathrm{CH}_{3}\right)_{3}\right) 1.8(4 \mathrm{H}, \mathrm{br}$, ring $\left.e q^{\mathrm{S}}\right), 0.3\left(4 \mathrm{H}, \mathrm{br}\right.$, ring $\left.a x^{\mathrm{C}}\right),-2.3\left(4 \mathrm{H}, \mathrm{br}\right.$, ring $\left.e q^{\mathrm{C}}\right),--5.8$ $(4 \mathrm{H}, \mathrm{br}, a c),-10.2(4 \mathrm{H}, \mathrm{br}, a c) ; \mathrm{m} / \mathrm{z}$ (MALDI+) $985(100 \%$, $[\text { EuL-2H] }]^{+}$), an appropriate isotope pattern was observed.

Europium(III) 1- $N$-(4-nitrophenyl)-4,7,10-tris-( $N$-ethylacetate)-1,4,7,10-tetraazacyclododecane-1,4,7,10-tetraacetamide triflate salt (Eu16). The complex was obtained as a yellow solid. ${ }^{1} \mathrm{H}$ NMR (400 $\left.\mathrm{MHz}, \mathrm{CD}_{3} \mathrm{CN}\right) \delta=27.4(4 \mathrm{H}$, br, ring $\left.a x^{\mathrm{S}}\right), 10.1(4 \mathrm{H}, \mathrm{m}, \mathrm{Ar}), 5.8\left(6 \mathrm{H}, \mathrm{m}, \mathrm{OCH}_{2}\right), 3.2(9 \mathrm{H}, \mathrm{m}$, $\left.\mathrm{CH}_{3}\right), 1.8\left(4 \mathrm{H}\right.$, br, ring $\left.e q^{\mathrm{S}}\right), 0.2\left(4 \mathrm{H}\right.$, br, ring $\left.a x^{\mathrm{C}}\right),-2.4(4 \mathrm{H}, \mathrm{br}$, ring $\left.e q^{\mathrm{C}}\right),-6.0(4 \mathrm{H}, \mathrm{br}, a c),-10.4(4 \mathrm{H}, \mathrm{br}, a c) ; \mathrm{m} / \mathrm{z}$ (MALDI+) $929\left(100 \%,[\mathrm{EuL}-2 \mathrm{H}]^{+}\right)$an appropriate isotope pattern was observed.

Europium(III) 1- $N$-(4-aminophenyl)-4,7,10-tris-( $N$-ethylacetate)-1,4,7,10-tetraazacyclododecane-1,4,7,10-tetraacetamide triflate salt (Eu17). $10 \%$ palladium on carbon $(10 \mathrm{mg})$ was added to a solution of Eu10 (42 mg, $45 \mu \mathrm{mol})$ in ethanol $(10 \mathrm{~mL})$ and the flask was flushed with $\mathrm{N}_{2}$ for $30 \mathrm{~min}$. The reaction mixture was then shaken on a Parr hydrogenator under a hydrogen pressure of 40 psi at room temperature for 3 hours. The solution was filtered and the solvents removed under vacuum to afford the title complex as a colorless solid in quantitative yield.

${ }^{1} \mathrm{H}$ NMR (400 MHz, $\left.\mathrm{CD}_{3} \mathrm{CN}\right) \delta=24.9\left(4 \mathrm{H}\right.$, br, ring $\left.a x^{\mathrm{S}}\right), 7.8$ $(2 \mathrm{H}, \mathrm{m}, \mathrm{Ar}), 7.5(2 \mathrm{H}, \mathrm{m}, \mathrm{Ar}), 5.92\left(6 \mathrm{H}, \mathrm{m}, \mathrm{OCH}_{2}\right), 3.4(9 \mathrm{H}, \mathrm{m}$, $\left.\mathrm{CH}_{3}\right), 1.5\left(4 \mathrm{H}\right.$, br, ring $\left.e q^{\mathrm{S}}\right), 0.4\left(4 \mathrm{H}, \mathrm{br}\right.$, ring $\left.a x^{\mathrm{C}}\right),-2.2(4 \mathrm{H}, \mathrm{br}$, ring $\left.e q^{\mathrm{C}}\right),-8.22(4 \mathrm{H}, \mathrm{br}, a c),-12.4(4 \mathrm{H}, \mathrm{br}, a c) ; \mathrm{m} / \mathrm{z}$ (MALDI+) $899\left(100 \%,[\mathrm{EuL}-2 \mathrm{H}]^{+}\right)$an appropriate isotope pattern was observed. 